\title{
Prenatal testing in Huntington disease: after the test, choices recommence
}

\author{
Hanane Bouchghoul $^{1}$, Stéphane-Françoise Clément ${ }^{2,3}$, Danièle Vauthier ${ }^{1}$, Cécile Cazeneuve ${ }^{4}$, Sandrine Noel ${ }^{4}$, \\ Marc Dommergues $^{1}$, Delphine Héron ${ }^{4}$, Jacky Nizard ${ }^{1}$, Marcela Gargiulo ${ }^{4,5,6,7}$ and Alexandra Durr ${ }^{\star, 3,4,7}$
}

The objective of this study was (1) to determine the impact of prenatal diagnosis (PND) for Huntington disease (HD) on subsequent reproductive choices and family structure; and (2) to assess whether children born after PND were informed of their genetic status. Out of 354 presymptomatic carriers of HD gene mutation, aged 18-45 years, 61 couples requested 101 PNDs. Fifty-four women, 29 female carriers and 25 spouses of male carriers, accepted to be interviewed (0.6-16.3 years after the last PND, median 6.5 years) on their obstetrical history and information given to children born after PND. Women were willing to undergo two or more PNDs with a final success rate of $75 \%$. Reproductive decisions differed depending on the outcome of the first PND. If favourable, $62 \%$ couples decided against another pregnancy and $10 \%$ chose to have an untested child. If unfavourable, $83 \%$ decided for another pregnancy $(P<0.01)$, and the majority $(87 \%)$ re-entered the PND procedure. In contrast, after a second PND, only 37\% asked for a PND and 30\% chose to have an untested child. Thirty-three percent had both, tested and untested children. Among children born after PND, 10 years and older, $75 \%$ were informed of their genetic status. The decision to prevent transmission of the HD mutation is made anew with each pregnancy. Couples may need more psychological support after PND and pre-counselling sessions should take into account the effect of the outcome of a first PND on subsequent reproductive choices.

European Journal of Human Genetics (2016) 24, 1535-1540; doi:10.1038/ejhg.2016.59; published online 15 June 2016

\section{INTRODUCTION}

In autosomal dominant adult-onset diseases for which there are no curative treatments, persons at risk prefer not to transmit the disease to future generations. Huntington disease (HD) is one of the dominantly inherited adult-onset disorders for which prenatal diagnosis (PND) is available and, thus, the possibility of avoiding transmission of the mutation. Technically, parents have two options: (i) direct PND, during a spontaneous pregnancy, by analysis of fetal DNA obtained by chorionic villus sampling or amniocentesis followed by termination of pregnancy if the fetus carries the HD mutation. (ii) preimplantation genetic diagnosis (PGD), an alternative that exists since 1996. ${ }^{1}$ PGD involves analysis of an embryo obtained by in vitro fertilisation, which is followed by implantation of a non-carrier embryo if one is found. Success rates for having a child with this procedure are still low: $\sim 20 \%{ }^{2}$ Sperm or egg donations are also possible. Some parents accept the risk of transmitting the disease, others may renounce to have a child. Factors that can influence reproductive choices are numerous and complex. They include aversion to the risk of transmitting the disease and personal considerations regarding termination of pregnancies and assisted reproduction, as well as the future parents' understanding of the different options that are presented to them during counselling sessions.

HD is an autosomal dominant neurological disorder characterised by psychiatric manifestations, cognitive impairment and movement disorders. In the great majority of cases, the first symptoms appear between age 30 and 50 years, and the disease progresses chronically over decades. The huntingtin (HTT) gene, responsible for $>90 \%$ of HD phenotypes, contains a trinucleotide (CAG) repeat in its first exon; the mutation consists of an abnormal expansion of the repeat beyond a threshold of 35 units. The risk of transmitting the mutation is a priori $50 \%$ for each child. During transmission, the CAG repeat may expand, a phenomenon called anticipation, or contract slightly. ${ }^{3}$

Since the introduction of direct testing for HD in 1993, uptake for presymptomatic genetic testing by at-risk individuals remains infrequent: $5-25 \%$ of the population at risk for HD opt for the test; ${ }^{4-8}$ requests for PND are even less frequent (10-22\%) among mutation carriers. ${ }^{9-11}$ Acceptance and uptake of PND by individuals at risk for HD differ greatly from country to country. A collaborative European study analysed 191 couples requesting 305 PND (106 for exclusion testing). ${ }^{9}$ In addition, 46 couples were studied in Belgium ${ }^{12}$ and 126 and 132 couples in the Netherlands. ${ }^{11,13}$ The results showed low uptake of a first PND by carriers and complexity of the decisionmaking process for every pregnancy.

Here, we present results obtained in a cohort of HD carriers who requested a PND. The aim of the study was dual; (1) to determine the impact of PND on subsequent reproductive choices and family structure; and (2) to assess if children born after PND were informed about their genetic status.

\footnotetext{
${ }^{1}$ APHP, Department of Gynaecology-Obstetrics, University Hospital Pitié-Salpêtrière, Sorbonne Universities, Paris, France; ${ }^{2}$ Department of Research in Ethics, EA1610, University Paris Sud, Orsay, France; ${ }^{3}$ ICM (Institut du Cerveau et de la Moelle épinière), INSERM U1127, CNRS UMR7225, Sorbonne Universities - UPMC University Paris VI UMR_S1127, Paris, France; ${ }^{4}$ APHP, Department of Genetics, University Hospital Pitié-Salpêtrière, Paris, France; ${ }^{5}$ Laboratory of Clinical Psychology and Psychopathology, EA 4056, University Paris Descartes, Institute of Psychology, Paris, France; ${ }^{6}$ APHP, Institut de Myologie, University Hospital Pitié-Salpêtrière, de Paris, Paris, France

*Correspondence: Professor A Durr, ICM (Institut du Cerveau et de la Moelle épinière), INSERM U1127, CNRS UMR7225, Sorbonne Universities - UPMC University Paris VI UMR_S1127, Groupe Hospitalier Pitié-Salpêtrière, 47 boulevard de l'Hôpital 75651, Paris Cedex 13, France. Tel: +33 157274682 ; E-mail: alexandra.durr@icm-institute.org ${ }^{7}$ Co-last authors.

Received 4 November 2015; revised 29 April 2016; accepted 5 May 2016; published online 15 June 2016
} 


\section{PARTICIPANTS AND METHODS}

Among the 1577 individuals at risk for HD who requested presymptomatic testing in our centre between April 1996 and January 2015, 74\% $(n=1170)$ were 18-45 years old, ages favourable for reproduction. In this age group, 354 carried the mutation, and among them $17 \%(n=61)$ of the couples requested a PND in our centre. Pre-test counselling had been provided to all testees, including CAG repeat size categories and the uncertainty about correlation with age at onset.

General information and information concerning reproduction were recorded. Information on mutation carriers included gender, motivation for presymptomatic testing, age at the time of result disclosure, number of CAG repeats in the HTT gene. Information on the pregnancies included the gender of the carrier, maternal age, obstetrical history, result of the PND (number of CAG repeats), pregnancy outcome (termination of pregnancy, live birth, fetal loss).

Each women was contacted by phone. The researcher explained the aim of the study and ask if she would be inclined to answer a series of questions. The interviews were semi-structured and an interview schedule was used to structure interviews (Supplementary data S2).

The first part of the questionnaire covered the following items: number of pregnancies since the PND, how these pregnancies were obtained (same partner or not, spontaneous, PGD, egg or sperm donation), whether a PND was performed outside our centre, pregnancy outcome.

The second part concerned their subjective experience with PND procedures, family structure, impact of the disease, if already manifest, and the information given to children, born after a PND or not, on their carrier status.

The answers were coded thematically by the interviewers (HB and SC). Motivation for testing were categorized in 'need to know', 'planning to have a child', 'ongoing pregnancy', 'suspicion of symptoms', 'anticipation of the future', 'do not know'. Personal experiences of PND were categorized as 'positive' (good experience, difficult or painful experience but no regrets) or 'negative' (bad experience, regrets). Impact on the family was called it using the following categories: couple stability (same or different partner) and disclosure of the child's genetic status to the child (informed/not informed).

\section{Statistics}

We compared categories (motivation for presymptomatic testing and negative or positive comments on personal experience of PND) with a $\chi^{2}$-test (or Fisher's exact test when the numbers were too small). We compared means with $t$-test. Results were considered statistically significant if $P \leq 0.05$. Data were analysed with Stata 12 software (StataCorp LP, College Station, TX, USA).

\section{RESULTS \\ Participants}

Of the 61 women who had PNDs, 30 were mutation carriers and 31 spouses of carriers. Five spouses of male carriers were lost to follow-up. One spouse of a male carrier refused to respond, and one female carrier was not able to answer because of severe dysarthria.

The remaining 54 women form our database ( 29 female carriers, 25 spouses of male carriers). The baseline characteristics of female carriers and spouses of male carriers were similar (Table 1).

The median time lapse between presymptomatic testing result and a first PND was 1.4 years. The main individual motivations for presymptomatic testing were ongoing pregnancy or a plan to have a child (40\%) and the 'need to know' and 'to be relieved of uncertainty concerning their genetic status' (37\%). For $10 / 61$ couples (16\%), the motivation for presymptomatic testing was an ongoing pregnancy

Table 1 Characteristics of carriers of the Huntington Disease mutation or spouses of a carrier at the time of the first prenatal diagnosis and the interview

\begin{tabular}{|c|c|c|c|}
\hline At the time of the first PND $\mathrm{n}=61$ & Female carrier $\mathrm{n}=30$ & Spouses of a carrier $n=31$ & P-values \\
\hline Median age in years (range) & $27.2(24.8-31.4)(20-40)$ & $30.5(26.5-33.2)(21-30)$ & 0.15 \\
\hline Mean parity ${ }^{a}(n)$ & $0.13(0-1)$ & $0.35(0-3)$ & 0.11 \\
\hline Nulliparous $(n)$ & $87 \%(26)$ & $74 \%(23)$ & 0.20 \\
\hline One or more terminations $(n)$ & $13 \%(4)$ & $26 \%(8)$ & 0.33 \\
\hline One or more miscarriages $(n)$ & $13 \%(4)$ & $16 \%(5)$ & 0.76 \\
\hline Motive for presymptomatic testing $(n)$ & & & 0.62 \\
\hline Need to know & $43 \%(13)$ & $29 \%(9)$ & 0.24 \\
\hline Planning to have a child & $23 \%(7)$ & $23 \%(7)$ & 0.94 \\
\hline On-going pregnancy & $10 \%(3)$ & $23 \%(7)$ & 0.30 \\
\hline Suspicion of symptoms & $7 \%(2)$ & $13 \%(4)$ & 0.67 \\
\hline Anticipation of the future & $10 \%(3)$ & $10 \%(3)$ & 0.65 \\
\hline Unknown & $7 \%(2)$ & $3 \%(1)$ & 0.61 \\
\hline Median number of expanded CAG repeats in the HD gene & $42.0(40-58)$ & $45.040-53)$ & 0.09 \\
\hline At the time of the interview $\mathrm{n}=54$ & Female carrier $n=29$ & Spouses of a carrier $\mathrm{n}=25$ & P-value \\
\hline Median age in years (range) & $34.9(31.5-39.6)(24-52)$ & $30.5(26.5-33.2)(27-46)$ & 0.08 \\
\hline Mean parity $(n)$ & $1.2(0-2)$ & $1.4(0-4)$ & 0.49 \\
\hline Nulliparous $(n)$ & $17 \%(5)$ & $16 \%(4)$ & 0.90 \\
\hline One or more terminations ${ }^{b}(n)$ & $55 \%(13)$ & $56 \%(14)$ & 0.95 \\
\hline New pregnancy $(n)$ & $34 \%(10)$ & $24 \%(6)^{c}$ & 0.41 \\
\hline Marital status & & & 0.41 \\
\hline Same partner $(n)$ & $76 \%(22)$ & $60 \%(15)$ & \\
\hline Single $(n)$ & $21 \%(6)$ & $28 \%(7)$ & \\
\hline New partner $(n)$ & $3 \%(1)$ & $12 \%(3)$ & \\
\hline
\end{tabular}

(Q1-Q3) first and third quartiles.

a Number of previous pregnancies.

boluntary terminations.

cNew pregnancies with a new partner were not taken into account. 
(three women carrying the mutation and seven men). The median delay between the last PND in our centre and the interview was 6.5 years (6 months to 16.3 years).

Eighteen percent of the women (12/61) had one or more untested child(ren) before their first PND (11/12 had one child, 1/12 had 3). It was the first PND experience for all of them. At the time of the interview, female carriers were slightly older than the spouses (median age 34.9 versus 30.5 , NS).

A total of 100 pregnancies, including one twin pregnancy (dichorionic, diamniotic), led to 101 PNDs (96 by chorionic villus sampling and 5 by amniocentesis); 50 results were favourable, 51 unfavourable. The mean number of PNDs per couple was 1.6 (1-6).

The number of pathological CAG repeats ranged from 37 to 61 triplets in the fetuses and from 40 to 58 triplets in the carrier parent. The mean number in the fetus was 44 when the mother was the carrier $(37-53, n=22)$ and 47 when the carrier was the father (40-61, $n=23)$. As expected, larger expansions were more often observed when the father transmitted the mutation $(52 \%(+1$ to +11 additional repeats) versus $15 \%(+2)$ for the mothers, $P=0.02)$.

Out of 51 pregnancies with an unfavourable result, 49 were terminated, one resulted in a miscarriage and one in a live birth. For the twin pregnancy, the one carrying the mutation was terminated and the other one was continued to term. All 50 pregnancies with favourable PNDs led to live births at term.

One woman continued her pregnancy despite an unfavourable result. In fact, she discovered she was a carrier while pregnant and received the results of both the presymptomatic and the prenatal test within a short time frame. She decided not to terminate the pregnancy. So far, she has not disclosed to her oldest son (9 years old) the fact that he is a carrier.

Decision making after a favourable or unfavourable first PND Of the 29 (Figure 1) women with an unfavourable first PND, 24 had at least one subsequent pregnancy: 19 pregnancies with a PND, 1 with a PGD, 1 with sperm donation and 3 with no medical intervention.

Of the 26 women with a favourable first PND, 10 pregnancies followed: 8 had a PND, and 2 had no medical intervention. Overall, the subsequent pregnancy rate was significantly greater (83\%) among women with unfavourable PND results than among those with favourable results $(38 \%, P<0.01)$. There were no differences between women with favourable or unfavourable outcomes after PNDs (Supplementary Table S1).

After the last pregnancy with a PND, 20 women (37\%) had no children.

Women who requested two PNDs $(n=23)$ had nine subsequent pregnancies, including seven untested and two with PGD. Three women with three and more PNDs had five additional tested pregnancies (Table 2).

\section{Subjective experience with PND}

The women interviewed often expressed satisfaction at being contacted and talked about their PND experience. There was a direct link between their personal experience and the negative (unfavourable result) or positive (favourable result) issue of the procedure. The group who made negative comments $(20 / 53,38 \%)$ comprised a larger number of women who terminated the pregnancy medically $(13 / 20$, $65 \%$ versus $16 / 33,48 \%$ in the group making positive comments, $P=0.27$ ) and who reported no favourable outcome (no child born as a result of the PND: $8 / 20,40 \%$ versus $7 / 33,21 \%$ in the group making positive comments $P=0.21$ ). However, painful memories were mentioned by both groups for all women.

The choice not to transmit the mutated gene, even if it meant going through a difficult experience, was one of the most frequent positive arguments put forward. Some women declared that, even if they found it painful, they were grateful to have had the choice. 'I decided to have a child when I learned about the possibility of asking for PND. The result was hard to accept but everything went well; I am fine now' (female carrier with an unfavourable first but favourable second PND).

Some described the Cornelian conflict they faced when they had to choose between their own desire to give birth to a child and the fear that he or she would be confronted to HD later in life: 'If it had been for me I would have kept them, but I thought of them and I do not regret having gone through with it' (spouse with two unfavourable PNDs, no child). Some explained that they had thoughts of loss, death and disease during this painful period: 'It is very hard to think that the baby could be sick, to lose her' (female carrier with a favourable PND), 'During the first 3 months, it is difficult to live' (spouse with a favourable result); $4 / 18$ (22\%) said that they would not go through the experience again, and one indicated that she would have preferred a

\section{Women's decision after a $1^{\text {st }}$ favorable or unfavorable PND}
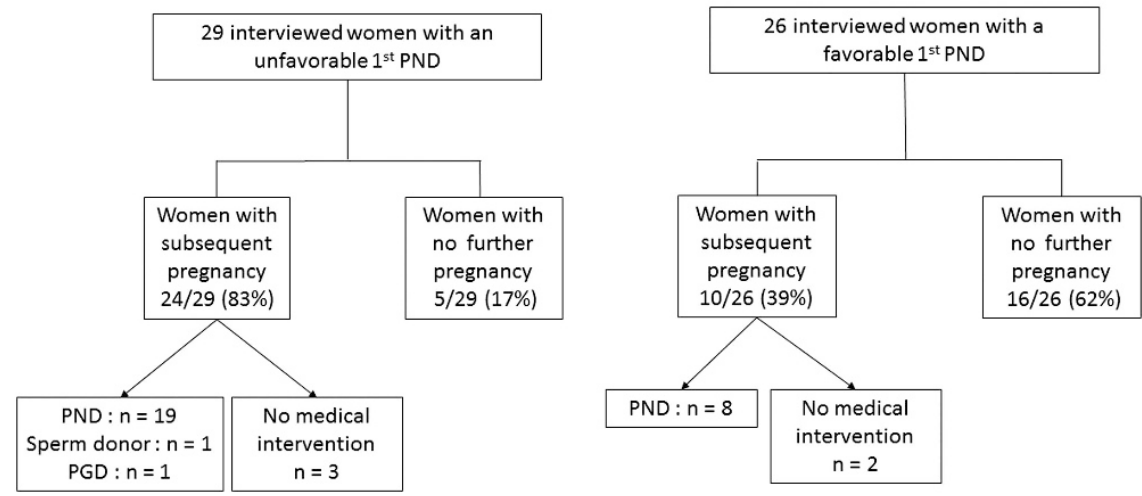

Figure 1 Women's decisions to subsequent pregnancies after the first prenatal diagnosis (PND) for Huntington Disease. We interviewed 54 women, 29 with a first unfavourable and 26 with a favourable first PND and showed the subsequent decisions: the subsequent pregnancy rate was significantly greater (83\%) among women with unfavourable PND results than among those with favourable results $(38 \%, P<0.01)$. 
PGD. Women who did renounce further pregnancies, considering this being the only way not to pass on the disease to their offspring, described the ensuing grief: 'It was painful, especially not to have children today' (spouse, one unfavourable PND, no child): 'I needed time to get over this experience. I have been contemplating the possibility of a pregnancy for 2 years now, but I don't know if it will be possible due to my age' (spouse, one unfavourable result, no child, new partner).

\section{Impact on the family structure}

At the time of the interview, the number of women who were no longer with the same partner or had changed partners was slightly, but not significantly, higher in the spouse group (40 versus $24 \%, P=0.41$ ) (Table 1). There was no influence of the final outcome of the PND(s), as $4 / 15(27 \%)$ women with no child born after PND were separated versus $13 / 39(33 \%)$ women with one or more child(ren) born after PND.

\section{Information given to children}

Thirty-nine of (Table 3 ) the interviewed women had one or more child(ren) $(n=48)$ subsequent to a PND. The median age of the children was 13 years, ranging from 3 months to 19 years. The total number of siblings in these 39 families was 70 . In $33 \%$ of the families having more than one child, not all children of the same sibship were born after a PND.
Twelve women (32\%) disclosed the child's genetic status to the child, the information was mostly transmitted by spouses (4/21 female carriers, $19 \%$ versus $8 / 17$ spouses, $47 \%, P=0.07$ ). One woman was excluded because her child was only 3 months old. The children received the information at a median age of 14 and 12 years (from 3 to 18 years of age) in the carrier and spouse groups, respectively. The median age of the children was 6.7 years old and it is worth noting that $50 \%(12 / 24)$ of the children above 6.7 were informed, versus $4 \%$ $(1 / 24)$ in the younger group. When the women explained why they informed the children, 6/9 answered that it was because their husbands or themselves had developed overt HD (2/4 female carriers, 50 versus $4 / 5$ spouses, 80\%). Mothers who did not inform their children indicated that either they would do so later $(10 / 13,77 \%$ : awaiting signs of the disease, too young) or they did not want to inform them $(3 / 13,23 \%$ : no interest for the child or too traumatic).

\section{DISCUSSION}

This is the first study about reproductive choices after a PND for HD and the information regarding their own status given to children born after PND. We interviewed only women because of the physical and personal impact of PND, even if the mutation carrier was the husband. The 61 couples that underwent 101 PNDs in our group represented $17 \%$ of carriers under 45 years of age who requested genetic counselling during the 18.5 -year period studied. This confirms the low uptake of prenatal testing for HD. ${ }^{9,14,15}$ There is a North-South

Table 2 Decisions regarding subsequent pregnancies after 1, 2 or 3 previous PND

\begin{tabular}{|c|c|c|c|c|}
\hline One $P N D \mathrm{n}=27 \mathrm{a} / 54$ result & $\begin{array}{c}\text { Number of } \\
\text { women }\end{array}$ & Women indicating subsequent pregnancy(ies) & $\begin{array}{c}\text { Children born } \\
\text { with no risk }\end{array}$ & $\begin{array}{l}\text { Children born } \\
\text { with } 50 \% \text { risk }\end{array}$ \\
\hline Fav. & 18 & 2 (3 pregnancies without PND) & 0 & $0^{b}$ \\
\hline Unf. & 10 & 5 (3 pregnancies without PND, 1 sperm donation, 1 PGD) & 2 & 3 \\
\hline \multicolumn{5}{|l|}{ Two PNDs $\mathrm{n}=23 / 54$ results } \\
\hline Fav./Fav. & 5 & 0 & 0 & 0 \\
\hline Fav./Unf. & 3 & 2 (each without PND) & 0 & 2 \\
\hline Unf./Fav. & 8 & 2 (each without PND) & 0 & $1^{\mathrm{c}}$ \\
\hline Unf./Unf. & 7 & 5 (3 pregnancies without PND2 PGD) & 2 & $4^{d}$ \\
\hline \multicolumn{5}{|l|}{ Three PNDs $\mathrm{n}=4 / 54$ results } \\
\hline Unf./Fav./Fav. & 1 & 0 & 0 & 0 \\
\hline Unf./Fav./Unf. & 2 & 2 (each with PND) & $1^{\mathrm{e}}$ & 0 \\
\hline Unf./ Unf;/Fav. & 1 & 1 (3 pregnancies with PND) & $2^{\mathrm{e}}$ & 0 \\
\hline
\end{tabular}

Abbreviations: Fav., favourable result; Unf., unfavourable result.

ancluding one twin pregnancy.

bTwo miscarriages and one ectopic pregnancy.

${ }^{b}$ One miscarriage.
cone miscariages

dOne women had two subsequent pregnancies without PND.

eOne pregnancy was terminated following an unfavourable result.

Table 3 Information given to children

\begin{tabular}{lcc}
\hline Couples with children born after a PND $\mathrm{n}=39$ & Female carrier $\mathrm{n}=22$ & Spouses $\mathrm{n}=17$ \\
\hline Number of children born after a PND & 27 & 21 \\
Total number of siblings & 36 & 34 \\
Disclosures of the child's genetic status to the child & $18 \%(4)$ & $47 \%(8)$ \\
Informed siblings & $17 \%(6)$ & $41 \%(14)$ \\
Age of children & $13(8-17)(3 \mathrm{~m}-18 \mathrm{y})$ & $13(9-15)(2 \mathrm{y}-19 \mathrm{y})$ \\
Age at which they were informed & $14(8-18)(3 \mathrm{y}-18 \mathrm{y})(N=4)$ & $12(9 \mathrm{y}-13 \mathrm{y})(N=7)$ \\
\hline
\end{tabular}


decrease in PND uptake; the number of PND per couple in the Netherlands was higher than in more southern populations $\left(2.8,1-9^{13}\right.$ versus $1.6,1-6)$. Interestingly, in our study the proportion of female and male carriers among those requesting PND was similar (50\%), whereas in other studies more male carriers were found. A possible explanation could be that women who are carriers of the mutation, are more prone to avoid the question of termination. ${ }^{11,13}$

\section{Impact of PND on decision making regarding subsequent pregnancies}

One of the main results of this study is that motivation for PND during a second pregnancy is much stronger after a previous unfavourable PND followed by termination of pregnancy $(65.5 \%)$ than after a favourable PND followed by the birth of a non-carrier child $(31 \%, P=0.01)$. This result could suggest that the couple's willingness to undergo PND is closely linked to the desire to have at least one child not at risk for HD. They rarely changed their strategy (8 women, 15\%) for the first two PNDs: if the first was unfavourable, they requested a second PND, and if the second was favourable, they choose to have no further pregnancies. However, after an unfavourable second PND most couples changed their strategy: they chose to risk having a child carrying the mutation (45\%), switch to PGD or abandoned the idea of having a child (27\%). Women explained that the delay between confirmation of the pregnancy and the result of the PND is a period of anguish and distress. Time stops while parents waited for the result, and they could not mentally invest in the future child because of fear of having to terminate the pregnancy. In at least one case, the guilt that accompanied termination of the pregnancy was so painful that it was impossible for the woman to consider a second PND. PGD allows them to avoid this difficulty, but the chances of having a child remain very low $(20 \%),{ }^{2}$ and the PGD procedure is long and heavily medicalized. The final success rate of PND was $75 \%$ in our cohort and, at the time of the interviews, $87 \%$ of the couples had progeny.

After their last PND, a large number of women choose not to take recourse to PND or other technical options for subsequent pregnancies; 12 children or fetuses (ongoing pregnancies) were thus at $50 \%$ risk for HD. This was unexpected. This has been explained by unwillingness to repeat the experience of a PND, reluctance to go through termination of the pregnancy and the hope that a treatment will be available with time. ${ }^{14}$ However, these reasons do not seem to apply to our cohort, as most women who had unfavourable results went through the difficult process a second time, although they had already lived through the invasive sampling procedure, the waiting, the announcement of an unfavourable result and termination of the pregnancy, sometimes at advanced gestational ages. However, the above-mentioned motivations might still be valid after the birth of a child, as couples did not contemplate a second pregnancy with PND if the first PND was favourable $(16 / 26,61 \%)$, or a third pregnancy if the second PND was favourable (10/16, 62.5\%).

When presymptomatic testing is decided during an ongoing pregnancy, the situation is very stressful because of the short delay between the decision to go through the procedure and disclosure of the result; this leaves little time for decision making. The number of pregnancies continued after an unfavourable PND in our cohort $(2 \%)$ was smaller than the $8-14 \%$ reported in studies in the Netherlands and Canada. ${ }^{13,14,16}$ This might reflect different attitudes towards pregnancy termination, ie, our counsellors insist on the fact that if the fetus's status would not change the couple's decision about the pregnancy, there is no indication of PND.
The positive perception of the phone interviews, in particular when the PND experience had been negative, may indicate that women could benefit from post-PND follow-up. This was clearly expressed by two of them during open discussion. This is already recommended in the case of presymptomatic testing ('If there has been no further contact within 1 month of the delivery of the test result, the counsellor should initiate the follow-up.'). ${ }^{17,18}$ Whatever the result of the PND, the waiting and the sampling procedure are very difficult to live through. Future DNA testing on fetal DNA circulating in the mother's blood will certainly help decrease this psychological burden.

\section{Impact of PND on family structure}

PND, even with an unfavourable outcome, does not seem to affect the stability of couples, because the divorce rate observed in the general population in France $\left(46 \%\right.$ in $\left.2014^{19}\right)$ reflects the same proportion. Still, there is a tendency for spouses to leave a carrier more frequently than for female carriers to leave their husband (40 versus 24\%). An effect of presymptomatic testing for HD on family structure was previously reported. ${ }^{20}$ However, the couples in our cohort do not reflect the general at-risk population, as they had planned to have a child with their partner, and we could speculate that they engage in a long-term relationship despite the threat of the disease.

\section{Information given to children}

To our knowledge, this is the first study to investigate the make-up of families with children born after PND. The number of couples who had children prior to PND $(12 / 61,18 \%)$ is in line with other European data (19-25\%). ${ }^{10,11,13}$ In 33\% of the families children were born both, before and after PNDs. Changes of strategy by the parents reflects the complexity of the choices they are faced with and the weight of different factors such as the desire to have a child, the psychological burden of the genetic risk and the disease in the family, the acceptance of medical technology and pregnancy termination, the age of the women and her eventual reproductive history (miscarriages, difficulty getting pregnant, for example). The pressure of time could also be an important element in their decision; the wish to spend as much time as possible with a child could possibly take priority over the hereditary risk. Several authors have concluded that the desire to have a child overrides any other concerns, in the sense that having a healthy child allows a normal life and compensates for the threat of the disease. ${ }^{21}$

One-third of the couples in our cohort informed their offspring of their risk. In total, 75\% of the informed children were between 10 and 18 years old.

Addressing the subject of HD seems difficult for the parents in all cases, even in the absence of a risk of developing HD. ${ }^{22-26}$ Interestingly, in the absence of signs of the disease in the parent, the children are usually not informed, and spouses are much more inclined to talk about HD than female carriers. Disclosing such knowledge within the family has two dimensions: informing the child about his or her genetic status necessarily reveals the status of the carrier parent and his future - or actual - disease. This might also explain the choice of parents to not inform a child. In addition, if other sibling(s) is(are) at risk, the parents are also confronted with the psychological impact of the information and its effect on the family.

\section{CONCLUSION}

In our study population, PND was a well-accepted reproductive option for persons at risk of developing HD. We showed that women were willing to undergo two or more PNDs with a final success rate having a child of $75 \%$. Interestingly, $10 \%(5 / 55)$ couples chose not to 
have another PND but to have an untested child instead, 30\% (7/23) after a second PND. This seemed contradictory to their original wish not to transmit the disease and negating their main reason to be tested presymptomatically.

It is understandable to change one's mind regarding testing children when the sacrifices outweigh the benefits. This may be mentioned in the pre-PND counselling process. We show that couples requesting PND wish to have a child not at risk for HD, and that women accepted to undergo two or more PNDs to reach this objective. After a second unfavourable result, the desire to have a child was still present, but the burden of the procedure seemed too heavy and they either accept having a child with a $50 \%$ risk of carrying the mutation or they abandoned the idea of having a child. It is not unusual for the parents to make different reproductive choices in successive pregnancies, so that the genetic status of some of their children is known, whereas others remain at $50 \%$ risk of HD. Communication about HD is difficult for families, and parents tend to wait for overt signs of the disease before informing their children; spouses are more proactive than mothers who carry the mutation. The results of this study should help genetic counsellors present the available reproductive options and their outcome in the face of HD. It would be of interest to extend this study to other countries and other diseases.

\section{CONFLICT OF INTEREST}

The authors declare no conflict of interest.

\section{ACKNOWLEDGEMENTS}

We thank all patients who participated in the study. Special thanks to Elodie Schaerer who helped with data handling.

1 Schulman JD, Black SH, Handyside A, Nance WE: Preimplantation genetic testing for Huntington disease and certain other dominantly inherited disorders. Clin Genet 1996; 49: 57-58.

2 Van Rij MC, De Rademaeker M, Moutou C et al: Preimplantation genetic diagnosis (PGD) for Huntington's disease: the experience of three European centres. Eur J Hum Genet 2012; 20: 368-375.

3 Semaka A, Kay C, Doty C et al: CAG size-specific risk estimates for intermediate allele repeat instability in Huntington disease. J Med Genet 2013; 50: 696-703.

4 Hayden MR: Predictive testing for Huntington's disease: the calm after the storm. Lancet Lond Eng/ 2000; 356: 1944-1945.

5 Goizet C, Lesca G, Dürr A: French Group for Presymptomatic Testing in Neurogenetic Disorders Presymptomatic testing in Huntington's disease and autosomal dominant cerebellar ataxias. Neurology 2002; 59: 1330-1336.
6 Tassicker RJ, Teltscher B, Trembath MK et al: Problems assessing uptake of Huntington disease predictive testing and a proposed solution. Eur J Hum Genet 2009; 17: 66-70.

7 Morrison PJ, Harding-Lester S, Bradley A: Uptake of Huntington disease predictive testing in a complete population. Clin Genet 2011; 80: 281-286.

8 Hawkins AK, Creighton S, Hayden MR: When access is an issue: exploring barriers to predictive testing for Huntington disease in British Columbia, Canada. Eur J Hum Genet 2013; 21: 148-153.

9 Simpson SA, Zoeteweij MW, Nys K et al: Prenatal testing for Huntington's disease: a European collaborative study. Eur J Hum Genet 2002; 10: 689-693.

10 Evers-Kiebooms G, Nys K, Harper P et al: Predictive DNA-testing for Huntington's disease and reproductive decision making: a European collaborative study. Eur J Hum Genet 2002; 10: 167-176.

11 van Rij MC, de Koning Gans P a. M, Aalfs CM et al: Prenatal testing for Huntington's disease in the Netherlands from 1998 to 2008. Clin Genet 2014; 85: 78-86.

12 Decruyenaere M, Evers-Kiebooms G, Boogaerts A et al: The complexity of reproductive decision-making in asymptomatic carriers of the Huntington mutation. Eur J Hum Genet 2007; 15: 453-462.

13 van Rij MC, de Koning Gans P a. M, van Belzen MJ et al: The uptake and outcome of prenatal and pre-implantation genetic diagnosis for Huntington's disease in the Netherlands (1998-2008). Clin Genet 2014; 85: 87-95.

14 Adam S, Wiggins S, Whyte P et al: Five year study of prenatal testing for Huntington's disease: demand, attitudes, and psychological assessment. J Med Genet 1993; 30: $549-556$.

15 Sizer EB, Haw T, Wessels T-M, Kromberg JGR, Krause A: The utilization and outcome of diagnostic, predictive, and prenatal genetic testing for huntington disease in johannesburg, South Africa. Genet Test Mol Biomark 2012; 16: 58-62.

16 Creighton S, Almqvist EW, MacGregor D et al: Predictive, pre-natal and diagnostic genetic testing for Huntington's disease: the experience in Canada from 1987 to 2000. Clin Genet 2003; 63: 462-475.

17 MacLeod R, Tibben A, Frontali $M$ et al: Recommendations for the predictive genetic test in Huntington's disease. Clin Genet 2013; 83: 221-231.

18 Guidelines for the molecular genetics predictive test in Huntington's disease: International Huntington Association (IHA) and the World Federation of Neurology (WFN) Research Group on Huntington's Chorea. Neurology 1994; 44: 1533-1536.

19 Divorces. Ined - Inst. Natl. D'études Démographiques. Available at https://www.ined.fr/ fr/tout-savoir-population/chiffres/france/mariages-divorces-pacs/divorces/ (accessed 22 Oct 2015).

20 Richards F, Williams K: Impact on couple relationships of predictive testing for Huntington disease: a longitudinal study. Am J Med Genet A 2004; 126 A: 161-169.

21 Richards FH, Rea G: Reproductive decision making before and after predictive testing for Huntington's disease: an Australian perspective. Clin Genet 2005; 67: 404-411.

22 Tyler A, Harper PS: Attitudes of subjects at risk and their relatives towards genetic counselling in Huntington's chorea. J Med Genet 1983; 20: 179-188.

23 Etchegary H: Discovering the family history of Huntington disease (HD). J Genet Couns 2006: 15: 105-117.

24 Dure LS, Quaid K, Beasley TM: A pilot assessment of parental practices and attitudes regarding risk disclosure and clinical research involving children in Huntington disease families. Genet Med Off J Am Coll Med Genet 2008; 10: 811-819.

25 Durr A, Hahn V, Gargiulo M et al: Analysis of the first 100 candidates for presymptomatic testing of Huntington's disease in Paris, France (abstract, Med. Genetik, 2, (H-9), 203. Berlin (Germany) 1995.

26 Gargiulo M, Capecchi T, Lahlou K, Evrard I, Feingold J, Durr A: Maladie dominante à révélation tardive: L'information du risque dans les familles (abstract). Marseille 2002.

Supplementary Information accompanies this paper on European Journal of Human Genetics website (http://www.nature.com/ejhg) 